\title{
Aminotic Fluid Index and its Correlation with Fetal Growth and Perinatal Outcome
}

\author{
Sonia Madaan ${ }^{1} \cdot$ Suman Lata Mendiratta $^{1} \cdot$ Pawan Kumar Jain $^{2} \cdot$ \\ Meenakshi Mittal ${ }^{1}$
}

Received: 25 March 2015/Accepted: 29 July 2015/Published online: 28 August 2015

(C) Society of Fetal Medicine 2015

\begin{abstract}
The present study aimed to measure amniotic fluid index (AFI) in high-risk pregnancies at $\geq 30$ weeks of gestation and its correlation with fetal growth and perinatal outcome. The Study was carried out in the Department of Obstetrics and Gynecology Hindu Rao Hospital, Delhi. Ultrasound examination was done on women with highrisk pregnancy (pregnancy-induced hypertension, intrauterine growth restriction, diabetes, and postdated pregnancy etc.) attending the antenatal OPD and maternity ward at $\geq 30$ weeks of gestation. AFI was measured by the four quadrant technique. The study included 48 (48\%) primigravida and $52(52 \%)$ multigravida. Sixteen patients with a history of previous abortion had mean AFI of $9.97 \mathrm{~cm}$ which was lower than patients with no history of previous abortion i.e., $11.87 \mathrm{~cm}$ ( $\mathrm{P} \geq 0.1$ insignificant). The mean AFI was maximum between 34-36 weeks, i.e., $13.1 \mathrm{~cm}$, after which, it gradually decreased to $9.08 \mathrm{~cm}$ beyond 40 weeks. Mean AFI of patients with $<40$ weeks of gestation gradually decreased from $12.2 \mathrm{~cm}$ to $8.0 \mathrm{~cm}$ after 42 weeks $(P$ value $<0.05$ ). Patients with IUGR had low mean AFI i.e., $9.8 \mathrm{~cm}$ whereas mean AFI in patients without IUGR was $12.1 \mathrm{~cm}$ (P value $<0.01$ ). Congenital anomalies were found in $4 \%$ patients in the present study. Out of seven cases of perinatal mortality encountered, four (57\%) had abnormal AFI values, of which, two had low AFI (i.e., $<8 \mathrm{~cm}$ ) and two had abnormally-high AFI values
\end{abstract}

Suman Lata Mendiratta

sumanmendi@yahoo.co.in

1 Obstetrics \& Gynaecology Department, North DMC Medical College \& Hindu Rao Hospital, A-3/224, Janakpuri, New Delhi 110058, India

2 Radiology Department, North DMC Medical College \& Hindu Rao Hospital, New Delhi, India i.e., $>18 \mathrm{~cm}$. Serious congenital malformations were present in three neonates, which resulted in early neonatal death. Out of these three, one patient had low AFI i.e., $<8 \mathrm{~cm}$ and one had abnormally-high AFI value i.e., $>24 \mathrm{~cm}$. The study supports the view that AFI is a quick, noninvasive, and good indicator of fetal outcome in high-risk pregnancy.

Keywords Amniotic fluid index (AFI) .

Oligohdramnios · Polyhdramnios · IUGR · Perinatal outcome $\cdot$ High-risk pregnancy

\section{Introduction}

Amniotic fluid acts as a protective layer which exerts a cushion-like effect for the growing fetus against mechanical and biological injury. Amniotic fluid may be regarded as the largest part of the fetal extracellular space, and it provides a more accessible means than fetal blood for investigation of the fetus and its environment. Amniotic fluid assessment is an integral part of the antenatal evaluation of pregnancies at risk for an adverse pregnancy outcome especially in the third trimester [1,2]. Detecting the fetus at risk for in utero damage or death, quantifying, and balancing the fetal risk against the risk of neonatal complications from immaturity, and determining the optimal time and mode of intervention are the cornerstone of modern day obstetrics care and perinatal medicine [3].

Reduced amniotic fluid volume (AFV) is associated with adverse effects such as meconium staining, congenital anomalies, growth retardation, dysmaturity, and fetal asphyxia [4-6]. Polyhydramnios is sometimes associated with major fetal anomalies [5], aneuploidy, macrosomia, and stillbirth [6]. Decreased AFV in those pregnancies 
without premature rupture of the membranes can reflect a fetus in chronic stress, shunting of blood to its brain, adrenal and heart and away from other organs, which include the kidney, and results in decreased fetal renal perfusion and urinary output. Phelan et al. [7] have recommended that labor induction be considered in patients with oligohydramnios (AFI $<5 \mathrm{~cm}$ ) to reduce the increased risk of fetal death and morbidity. An antepartum amniotic fluid index (AFI) of $5 \mathrm{~cm}$ or less is a predictor of adverse perinatal outcome in terms of meconium staining, cesarean section for fetal distress, birth weight, low Apgar scores and cord $\mathrm{pH}$ [8]. AFI of $>18 \mathrm{~cm}$ is taken as cut-off point for normal limit of AFI. Pregnancy complicated with polyhydramnios is also classified as high risk. AFV can be measured by dye-dilution techniques and by direct quantification at the time of cesarean delivery, but both methods are invasive, require laboratory support, and when measured at the time of operative abdominal delivery, cannot be used serially to evaluate high-risk pregnancies [1]. The limitation of the direct AFV measurement led to the use of ultrasonic AFV estimation. Ultrasonography is noninvasive and can clinically quantitate the AFV. There are various reported ultrasonographic modalities to assess AFV like (1) single deepest pocket (2) 2-diameter pocket, and (3) AFI by 4-quadrant method. Since the introduction of AFI by Phelan et al. [7], a rapid semi-quantitative assessment of AFV can be performed quickly, is easily taught, and is reproducible.

AFI also facilitated the diagnosis of a congenital anomaly not detected in referring clinics. The antepartum information regarding malformation was valuable for the patients and their health-care providers in deciding on the timing and mode of delivery, determining an etiology of intrauterine or postnatal death, and counseling of future pregnancies. The present study was aimed to measure AFI in high-risk pregnancies $\geq 30$ weeks of gestation and its correlation with fetal outcome.

\section{Materials and Methods}

This was a one year prospective study undertaken in the department of Obstetrics and Gynecology, Hindu Rao Hospital, Delhi a period of one year, after obtaining ethical clearance from institutional committee. Ultrasound examination was done on women with high-risk pregnancy (pregnancy-induced hypertension, intrauterine growth restriction, diabetes, and postdated pregnancy etc.) attending the antenatal OPD and maternity ward at $\geq 30$ weeks of gestation. Only patients who were sure of dates were included in the study. Patients with premature rupture of membranes and twin pregnancy were excluded from the study.
A detailed history was taken and a general physical and obstetrics examination were done. The patient was then subjected to ultrasonographic examination. Routine scan for fetal well being was done and AFI was measured by the 4-quadrant technique [7]. The vertical diameter of the maximum pocket was measured in centimeters in each of the four quadrants and measurement obtained from each quadrants were summed to form the AFI in centimeters.Follow-up of these patients was done till delivery and their neonatal outcome was noted in terms of fetal distress in labor, birth weight, Apgar at five minutes, congenital anomalies, and perinatal mortality. The results were compiled and analyzed.

\section{Results}

The present study was conducted on 100 antenatal patients over a period of one year. All the 100 patients included in the study had attended antenatal OPD/maternity ward at Hindu Rao Hospital and delivered at the Labor Room in Hindu Rao Hospital and the results were analyzed as follows.

The majority of cases i.e., $62(62 \%)$ were in the age group of 21-25 years, followed by $27(27 \%)$ in the age group 26-30 years, and the least were among the age group $>31$ years $(2 \%)$. The mean age for the entire study population was 24.31 years. The mean AFI for all age groups was $11.567 \pm 5.364 \mathrm{~cm}$. Present study included 48 (48\%) primigravida and $52(52 \%)$ multigravida.

The maximum cases i.e., $36(36 \%)$ had a gestational age between 36 to 38 weeks at the time of enrollment. The mean AFI was maximum, between 34-36 weeks i.e., $13.1 \mathrm{~cm}$, after which, it gradually decreased to $9.08 \mathrm{~cm}$ beyond 40 weeks (Table 1). Mean AFI of patients with $<40$ weeks of gestation gradually decreased from $12.2 \mathrm{~cm}$ to $8.0 \mathrm{~cm}$ after 42 weeks $(\mathrm{P}$ value $<0.05$ ) implies significant relationship between post-term pregnancy and AFI.

Antenatal complications in the present study group show that anemia $(\mathrm{Hb}<10 \mathrm{~g} \%)$ was the commonest complication found in $27 \%$ cases, followed by IUGR (24\%), PIH (20\%), previous abortions (16\%) cases, previous lower segment cesarean section $(11 \%)$, oligohydramnios (8\%), post-term pregnancy (6\%), and polyhydramnios (5\%) being the next common complications (Table 2).

Mean AFI of patients with IUGR was $9.8 \mathrm{~cm}$ whereas that in patients without IUGR was $12.1 \mathrm{~cm}$, implies a highly-significant relationship between IUGR and AFI. It was found that out of 20 patients who were hypertensive, 8 (40\%) had low AFI i.e., $<8 \mathrm{~cm}$. The mean AFI of hypertensive patients was $10.7 \mathrm{~cm}$ which was not significantly different, statistically, from mean AFI of 
Table 1 Distribution of patients according to gestation age at enrollment

\begin{tabular}{lccc}
\hline POG at enrollment (weeks) & No. of patients & Mean AFI $(\mathrm{cm})$ & SD \\
\hline $30-32$ & 1 & 4.000 & - \\
$32-34$ & 4 & 9.200 & 1.860 \\
$34-36$ & 15 & 13.133 & 7.067 \\
$36-38$ & 36 & 12.908 & 5.602 \\
$38-40$ & 24 & 11.354 & 4.977 \\
$>40$ & 20 & 9.085 & 2.948 \\
Total & 100 & 11.567 & 5.364 \\
\hline
\end{tabular}

$A F I$ amniotic fluid index, $P O G$ period of gestation, $S D$ standard deviation

Table 2 AFI in various high-risk pregnancy groups (some cases had more than one high-risk factor)

\begin{tabular}{lcccr}
\hline High-risk pregnancy factor & No. of cases (out of 100) & Mean AFI (cm) in cases & Mean AFI (cm) in controls & SD \\
\hline IUGR & 24 & 9.821 & 12.118 & 5.650 \\
Hypertension & 20 & 10.740 & 11.774 & 4.859 \\
Anemia & 27 & 11.637 & 11.541 & 2.690 \\
Previous LSCS & 11 & 11.864 & 11.530 & 6.581 \\
Post-term $>$ 40 wks & 20 & 9.085 & 12.188 & 2.948 \\
Diabetes & 5 & 12.300 & 11.528 & 5.709 \\
Rh-negative & 5 & 12.840 & 11.500 & 2.806 \\
Previous abortions & 16 & 9.97 & 11.870 & 2.617 \\
\hline
\end{tabular}

$A F I$ amniotic fluid index, IUGR intrauterine growth restriction, $L C S C$ lower segment cesarean section, $S D$ standard deviation

normotensive patients i.e., $11.7 \mathrm{~cm}$. The mean AFI in patients with anemia was $11.63 \mathrm{~cm}$ which was not significantly different from patients without anemia i.e., $11.54 \mathrm{~cm}$. There was no disparity between AFI in pregnancies with previous LSCS and non-LSCS group, mean AFI being 11.864 and 11.530 in cases and controls, respectively. Mean AFI of patients $<40$ weeks gradually decreased from $12.2 \mathrm{~cm}$ to $9.085 \mathrm{~cm}$ at $>40$ weeks and $8.0 \mathrm{~cm}$ after 42 weeks. There is no significant relation between diabetes mellitus and AFI, though mean AFI in pregnancies with diabetes $(12.3 \mathrm{~cm})$ was found to be more than those without diabetes $(11.5 \mathrm{~cm})$. Mean AFI in Rhnegative pregnancies was $12.8 \mathrm{~cm}$ which was slightly higher than rest of the cases i.e., $11.5 \mathrm{~cm}$. The mean AFI of 16 patients with a history of previous abortion was $9.97 \mathrm{~cm}$ which was lower than those with no history of previous abortions i.e., $11.87 \mathrm{~cm}$.
Table 3 depicts the relation of clinical assessment of liquor to the AFI obtained ultrasonographically. Mean AFI of 65 patients with clinically-normal liquor was $11.9 \mathrm{~cm}$; mean AFI of 27 patients with clinically-less liquor was $6.7 \mathrm{~cm}$ and mean AFI of eight cases with clinically-more liquor was $24.3 \mathrm{~cm}$. Statistical tests were applied and it was found that clinical estimation of liquor has a sensitivity of $74.35 \%$ and a specificity of $90.16 \%$, the accuracy being $84 \%$. Table 4 depicts AFI in relation to delivery outcomes.

\section{Discussion}

The present study was undertaken to measure AFI in highrisk pregnancies and to correlate it with fetal outcome. The mean maternal age in this study was $24.31 \pm 3.19$ years. Magann et al. [9, 10] in 1999 and 1997 reported the mean
Table 3 Comparison of AFI and clinical assessment of liquor (by palpation)

\begin{tabular}{lcccc}
\hline Clinical assessment of liquor & No. of cases & $\%$ & Mean AFI & SD \\
\hline Normal & 65 & 65 & 11.975 & 3.417 \\
Less & 27 & 27 & 6.785 & 1.789 \\
More & 8 & 8 & 24.388 & 3.272 \\
Total & 100 & 100 & 11.567 & 5.364 \\
\hline
\end{tabular}

$\mathrm{P} \leq 0.001$ (very highly significant) 
Table 4 AFI in relation to delivery outcomes

\begin{tabular}{|c|c|c|c|c|}
\hline \multicolumn{5}{|c|}{ Mode of delivery in relation to $\mathrm{AFI}^{\mathrm{a}}$} \\
\hline Mode of delivery & No. of cases & $\%$ & Mean AFI (cm) & SD \\
\hline Normal & 63 & 63 & 12.162 & 4.999 \\
\hline Forceps & 8 & 8 & 9.663 & 5.808 \\
\hline Cesarean & 29 & 29 & 10.800 & 5.959 \\
\hline Total & 100 & 100 & 11.567 & 5.364 \\
\hline \multicolumn{5}{|c|}{ Labor (spontaneous or induced) in relation to $\mathrm{AFI}^{\mathrm{b}}$} \\
\hline Labor & No. of cases & $\%$ & Mean AFI & SD \\
\hline Spontaneous & 54 & 98.35 & 11.976 & 4.385 \\
\hline Induced & 25 & 31.64 & 11.024 & 6.255 \\
\hline Total & 79 & 100 & 11.675 & 5.030 \\
\hline
\end{tabular}

AFI in relation to meconium staining of liquor ${ }^{c}$

\begin{tabular}{lcccc}
\hline Meconium staining of liquor & No. of cases & $\%$ & Mean AFI \\
\hline Absent & 85 & 85 & 11.754 & 5.020 \\
Present & 15 & 15 & 10.507 & 7.131 \\
Total & 100 & 100 & 11.567 & 5.364 \\
\hline
\end{tabular}

Relation of AFI with fetal heart rate ${ }^{\mathrm{d}}$

\begin{tabular}{lcccc}
\hline FHR & No. of cases & $\%$ & Mean AFI & SD \\
\hline Normal & 84 & 84 & 11.429 & 4.841 \\
Bradycardia & 12 & 12 & 11.975 & 7.507 \\
Tachycardia & 4 & 4 & 13.250 & 9.465 \\
Total & 100 & 100 & 11.567 & 5.364
\end{tabular}

AFI and its relation to birth weight ${ }^{\mathrm{e}}$

\begin{tabular}{|c|c|c|c|c|}
\hline Birth weight (g) & $\mathrm{N}$ & $\%$ & Mean AFI & SD \\
\hline$<2000$ & 17 & 17 & 7.976 & 2.467 \\
\hline $2001-2500$ & 27 & 27 & 12.448 & 6.526 \\
\hline $2501-3000$ & 43 & 43 & 12.247 & 4.106 \\
\hline$>3000$ & 13 & 13 & 12.185 & 7.476 \\
\hline Total & 100 & 100 & 11.567 & 5.364 \\
\hline \multicolumn{5}{|c|}{ Apgar $(5 \min )$ versus $A F I^{f}$} \\
\hline Apgar & $\mathrm{N}$ & $\%$ & Mean AFI & SD \\
\hline$<9$ & 10 & 10 & 11.550 & 8.448 \\
\hline $9-10$ & 90 & 90 & 11.569 & 4.976 \\
\hline Total & 100 & 100 & 11.567 & 5.364 \\
\hline
\end{tabular}

Congenital anomalies in the study group ${ }^{\mathrm{g}}$

\begin{tabular}{lrr}
\hline Congenital anomaly & Number & AFI $(\mathrm{cm})$ \\
\hline Bilateral CTEV & 1 & 6.3 \\
Osteogenetis imperfecta & 1 & 7.0 \\
Fetal ascites + treacheoesophageal interval + laryngeal stenosis & 1 & 26.0 \\
Tracheoesophageal fistula + anal agenesis & 1 & 10.0 \\
\hline
\end{tabular}


Table 4 continued

AFI versus perinatal mortality ${ }^{\text {h }}$

\begin{tabular}{llll}
\hline Perinatal mortality & AFI $(\mathrm{cm})$ & & \\
\hline & $<8$ & $8-18$ & $>18$ \\
Fresh stillbirth & - & - & 1 \\
Neonatal death & 2 & 3 & 1 \\
\hline
\end{tabular}

$A F I$ amniotic fluid index, CTEV congenital talipes equinovarus, $F H R$ fetal heart rate, $S D$ standard deviation

${ }^{a}$ Mean AFI in patients undergoing assisted delivery was found to be lower $(9.66 \mathrm{~cm}$ in forceps and $10.8 \mathrm{~cm}$ in cesarean group) than mean AFI in patients undergoing normal delivery (i.e., $12.16 \mathrm{~cm}$ ). It was also found that among 28 patients who had low AFI, 6 patients (42\%) had forceps delivery whereas $13(46.42 \%)$ had cesarean section

b The commonest cause of induction of labor in the study group was PIH (32\%), IUGR (16\%) and post-dated pregnancy (12\%)

${ }^{\mathrm{c}} \mathrm{P} \geq 0.1$. The mean value of AFI $(10.5 \mathrm{~cm})$ in patients with meconium staining of liquor was not significantly different from those without meconium staining of liquor $(11.7 \mathrm{~cm}) . \mathrm{P} \geq 0.1$ (not significant)

${ }^{\mathrm{d}} \mathrm{P} \leq 0.1$ (not significant). There was no correlation between the FHR and AFI. Out of 28 patients with low AFI, six (21.42\%) had fetal bradycardia and two $(7.14 \%)$ had fetal tachycardia

${ }^{\mathrm{e}} \mathrm{P} \leq 0.05$ (significant relation). Mean AFI was found to be significantly lower $(7.9 \mathrm{~cm})$ in low birth weight group $<2000 \mathrm{~g}$ (P value $\left.<0.05\right)$, implies significant relation between birth weight and AFI

${ }^{\mathrm{f}} \mathrm{P} \geq 0.1$ (not significant). Mean AFI in patients with APGAR $<9$ was not found to be significantly different from mean AFI in patients with Apgar $>9$

g Congenital anomalies were found in $4 \%$ patients in our study. The salient feature noted was that three (75\%) out of these four cases had abnormal AFI scores, two cases had low AFI scores $(<8 \mathrm{~cm})$ and one had high AFI value $(>24 \mathrm{~cm})$ while mean AFI of the remaining study group was $11.54 \mathrm{~cm}$

${ }^{\text {h }}$ Out of seven cases of perinatal mortality encountered in our study, four (57\%) had abnormal AFI values, of which two had low AFI (i.e., $<8 \mathrm{~cm}$ ) and two had abnormally-high AFI values (i.e., $>18 \mathrm{~cm}$ ). Three neonates had serious congenital malformations which resulted in early neonatal death, of which one patient had low AFI (i.e., $<8 \mathrm{~cm}$ ) and one had abnormally-high AFI value (i.e., $>24 \mathrm{~cm}$ )

maternal age of $24.8 \pm 5.3$ and $24.5 \pm 0.5$ years, respectively, which was comparative to our study. In our study parity has no relation to the distribution of cases as was seen in the study conducted by Magann et al. [10].

In our study anemia was the commonest complication, seen in $27(27 \%)$. Other significant complications were IUGR (24\%), PIH (20\%), previous LSCS (11\%), oligohydramnios $(8 \%)$, post-term pregnancy $(6 \%)$, and polyhydramnios (5\%). Magann et al. [11] reported IUGR in $4.74 \%$, PIH $3.16 \%$, and post-term pregnancy in $24.65 \%$ patients. Golan et al. [12] reported IUGR in $24.5 \%$ and hypertension in $22.1 \%$. In our study, among $27 \%$ cases that had anemia, no correlation was found between anemia and AFI.

In our study, out of 29 patients with AFI $<8 \mathrm{~cm}, 12$ (41.4\%) patients had IUGR. O'Brien et al. [13] concluded that fetal growth retardation is significantly associated with an AFI of $7 \mathrm{~cm}$, or less $(\mathrm{P} \leq 0.001)$.

In the present study, $20(20 \%)$ patients were hypertensive. Out of these patients, eight $(40 \%)$ had low AFI scores $(<8 \mathrm{~cm})$. In a study conducted by O'Brien et al. [13], out of 14 patients of mild PIH, only two patients i.e., $14 \%$ had a low AFI score $(\leq 7 \mathrm{~cm})$. In a study by Magann et al. [14] on patients with AFI $<5 \mathrm{~cm}, 7.16 \%$ patients were found to be hypertensive.

In this study, out of six patients with post-term pregnancy, oligohydramnios (AFI $<5 \mathrm{~cm}$ ) was seen in 2
(33.3\%) cases. In a study conducted by Marks and Divon [14] on post-term pregnancy, oligohydramnios was demonstrated in $59(11.5 \%)$ patients. If diabetes is associated with macrosomia and neural tube defects, polyhydramnios may develop. In our study, it was observed that mean AFI of five patients with diabetes was more $(12.3 \mathrm{~cm})$ than nondiabetic patients $(11.5 \mathrm{~cm})$ though it was not statistically significant ( $\mathrm{P}$ value $\geq 0.1$ ).

In the present study, AFI according to gestational age was studied. The mean AFI was $11.567 \mathrm{~cm}$. Maximum AFI of $13.13 \mathrm{~cm}$ (mean) was seen at 34-36 weeks of gestation, which gradually decreased to 9.085 (mean) after 40 weeks. Similarly, Phelan et al. [7] demonstrated a mean AFI of $12.9 \pm 4.6$ in 353 pregnancies at the gestational age ranging from 36-42 weeks. Bowen-Chatoor and Kulkarni [15] demonstrated a mean AFI of $9.93 \pm 4.37 \mathrm{~cm}$ at 42 weeks, which is comparable to our study.

In our study, it was observed that mean AFI of five patients with Rh-negative pregnancy was more i.e., $12.84 \mathrm{~cm}$ than rest of patients i.e., $11.5 \mathrm{~cm}$. Mean maternal weight in our study was $64.87 \pm 9.66 \mathrm{~kg}$, with majority of cases between $50-65 \mathrm{~kg}$. Fifty four $(68.35 \%)$ patients had spontaneous onset of labor while $25(31.64 \%)$ were induced. The main indication for induction was PIH, IUGR followed by postdated pregnancy.

In this study, out of 29 patients with low AFI $(<8 \mathrm{~cm})$, six $(20.7 \%)$ had forceps delivery, whereas $13(44.8 \%)$ 
patients had cesarean section. Out of 61 patients with normal AFI, 47 (77 \%) had normal delivery. According to Rutherford et al. [16], incidence of cesarean section was $58 \%$ in patients with AFI $<8 \mathrm{~cm}$ which is slightly more than our study. Their cesarean section rate in patients with AFI $>18 \mathrm{~cm}$ was $41 \%$, which was low $(30 \%)$ in our study.

In our study, 15 cases had meconium-stained liquor, out of which eight $(53.33 \%)$ had low AFI scores $(\leq 8 \mathrm{~cm})$. In a study by Golan et al. [12], a high incidence of meconium staining of liquor $(29.1 \%)$ was seen in oligohydramnios. In another study by Magann et al. [11], meconium-stained amniotic fluid was seen in $13.8 \%$ patients with AFI $\leq 5 \mathrm{~cm}$. In our study, out of eight patients with low AFI $(\leq 5 \mathrm{~cm})$, two $(25 \%)$ had fetal bradycardia. In a similar study by Magann et al. [11], it was found that in patients with AFI $<5 \mathrm{~cm}, 24 \%$ patients had fetal bradycardia, which was comparable to our study.

In our study, out of 29 patients with low AFI $(\leq 8 \mathrm{~cm})$, $17(58.62 \%)$ had low birth weight babies $(\leq 2500 \mathrm{~g})$. In 10 patients with AFI $>18 \mathrm{~cm}$, five $(50 \%)$ had low birth weight babies. Martinez-Frias et al. [17] also observed similar results in their study. According to Chauhan et al. [18], AFI of $<5 \mathrm{~cm}$ is associated with an increased risk of low $(<7)$ Apgar score.

In our study, four patients had babies with congenital anomalies, out of which, two $(50 \%)$ had low AFI $<8 \mathrm{~cm}$ and one had AFI $>18 \mathrm{~cm}$. In the study conducted by Martinez- Frias et al. [17] on malformed newborns, $3.01 \%$ had oligohydramnios. Renal anomalies and lung defects were associated with oligohydramnios. They reported polyhydramnios in $3.69 \%$ cases in their study. Esophageal and gastrointestinal anomalies, neural tube defects, and other central nervous system malformations were associated with polyhydramnios. In our study, osteogenesis imperfecta and bilateral CTEV were associated with low AFI of $<8 \mathrm{~cm}$, and tracheoesophageal fistula was associated with AFI $>18 \mathrm{~cm}$.

In our study perinatal mortality rate was $7 \%$. Of these seven cases, three babies $(42.85 \%)$ had serious congenital malformations, two cases $(28.57 \%)$ had low AFI of $<8 \mathrm{~cm}$ and two cases $(28.57 \%)$ had AFI $>18 \mathrm{~cm}$. Biggio et al. [19] reported $6 \%$ perinatal mortality in their study of 370 women, which was comparable to our study.

\section{Conclusion}

Amniotic fluid provides a more accessible means than fetal blood for surveillance of the fetus and its environment. Amniotic fluid assessment is an integral part of the antenatal evaluation of pregnancies at risk for an adverse pregnancy outcome. Our study supports the view that AFI is a quick, noninvasive and good indicator of fetal outcome in high-risk pregnancy.

Funding None.

\section{Compliance with Ethical Standards}

Conflict of interest None.

\section{References}

1. Magann EF, Chauhan SP, Bofil JA, et al. Comaparability of the amniotic fluid index and single deepest pocket measurement in clinical practice. Aust N Z J Obstet Gynecol. 2003;43:75-7.

2. Kofinas A, Kofinas G. Differences in amniotic fluid pattern and fetal biometric parameters in third trimester pregnancies with and without diabetes. J Matern Fetal Neonatal Med. 2006;19(10): 633-8.

3. Manning FA. Antepartum fetal testing: a critical appraisal. Curr Opin Obstet Gynecol. 2009;21(4):348-52.

4. Moore TR. Amniotic fluid dynamics reflect fetal and maternal health and disease. Obstet Gynecol. 2010;116:759-65.

5. Hashimoto BE, Kramer DJ, Brennan L. Amniotic fluid volume: fluid dynamics and measurement technique. Semin Ultrasound CT MR. 1993;14:40-55.

6. Sarno AP Jr, Ahn MO, Phelan JP. Intrapartum amniotic fluid volume at term. J Reprod Med. 1990;35:719-23.

7. Phelan JP, Smith CV, Broussard Small M, et al. Amniotic fluid volume assessment using four quadrant technique in pregnancy between 36 and 42 weeks gestation. J Reprod Med. 1987;32: $540-2$.

8. Nageotte MP, Towers CV, Asat T, et al. Perinatal outcome with the modified biophysical profile. Am J Obstet Gynecol. 1994; 170(6):1672-6.

9. Magann EF, Nevils BG, Chauhan SP, et al. Low amniotic fluid volume is poorly identified in singleton and twin pregnancies using the $2 \times 2 \mathrm{~cm}$ pocket technique of the biophysical profile. South Med J. 1999;92:802-5.

10. Magann EF, Perry KG Jr, Chauhan SP, et al. The accuracy of ultrasound evalution of amniotic fluid volume in singleton pregnancies: the effect of operator experiences and ultrasound interpretative technique. J Clin Ultrasound. 1997;25:249-53.

11. Magann EF, Kinsella MI, Chauhan SP, et al. Does amniotic fluid index of $\leq 5 \mathrm{~cm}$ necessitate delivery in high-risk pregnancies? A case control study. Am J Obstet Gynecol. 1999; 180(6):1354-9.

12. Golan A, Lin G, Evron S, et al. Oligohydramnios: maternal complications and fetal outcome in 145 cases. Gynecol Obstet Invest. 1994;37(2):91-5.

13. O'Brien JM, Mercer BM, Friedman SA, et al. Amniotic fluid index in hospitalized hypertensive patients managed expectantly. Obstet Gynecol. 1993;82:247-50.

14. Marks AD, Divon MY. Longitudinal study of the amniotic fluid index in post date pregnancy. Obstet Gynecol. 1992; 79:229-33.

15. Bowen-Chatoor JS, Kulkarni SK. Amniotic fluid index in the management of the postdates pregnancy. West Indian Med J. 1995;44(2):64-6. 
16. Rutherford SE, Phelan JP, Smith CV, et al. The four-quadrant assessment of amniotic fluid volume: an adjunct to antepartum fetal heart rate testing. Obstet Gynecol. 1987;70:353-6.

17. Martinez-Frias ML, Bermejo E, Rodriguez-Pinilla E, et al. Maternal and fetal factors related to abnormal amniotic fluid. J Perinatol. 1999;19(7):514-20.
18. Chauhan SP, Roberts WE, Martin JN Jr, et al. Amniotic fluid index in normal pregnancy: a longitudinal study. J Miss State Med Assoc. 1999;40(2):221-4.

19. Biggio JR, Wenstrom KD, Dubard MB, et al. Hydramnios prediction of adverse perinatal outcome. Obstet Gynecol. 1999;94:773-7. 